\title{
Considering the role of radiation therapy for gastrointestinal stromal tumor
}

This article was published in the following Dove Press journal:

OncoTargets and Therapy

12 May 2014

Number of times this article has been viewed

\section{Kimberly S Corbin' \\ Hedy L Kindler ${ }^{2}$ \\ Stanley L Liauw ${ }^{3}$}

'Department of Radiation Oncology, Memorial Medical Center, Springfield, IL, USA; ${ }^{2}$ Section of Hematology/ Oncology, Department of Medicine, University of Chicago, Chicago, IL, USA; ${ }^{3}$ Department of Radiation and Cellular Oncology, University of Chicago, Chicago, IL, USA
Correspondence: Stanley L Liauw Department of Radiation and Cellular Oncology, University of Chicago,

5758 S Maryland Ave,

MC 9006, Chicago, IL, USA

Tel +I 7737026870

Fax +I 7738347340

Email sliauw@radonc.uchicago.edu

\begin{abstract}
Gastrointestinal stromal tumors (GISTs) are rare mesenchymal tumors arising in the gastrointestinal tract. Over the last decade, the management and prognosis of GISTs has changed dramatically with molecular characterization of the $c$-kit mutation and the adoption of targeted systemic therapy. Currently, the standard of care for resectable tumors is surgery, followed by adjuvant imatinib for tumors at high risk for recurrence. Inoperable or metastatic tumors are treated primarily with imatinib. Despite excellent initial response rates, resistance to targeted therapy has emerged as a common clinical problem, with relatively few therapeutic solutions. While the treatment of GISTs does not commonly include radiotherapy, radiation therapy could be a valuable contributing modality. Several case reports indicate that radiation can control locally progressive, drug-resistant disease. Further study is necessary to define whether radiation could potentially prevent or delay the onset of drug resistance, or improve outcomes when given in combination with imatinib.
\end{abstract}

Keywords: GIST, imatinib, radiotherapy

\section{Background}

With an estimated 4,000-6,000 cases per year in the US, ${ }^{1}$ gastrointestinal stromal tumors (GISTs) are the most common mesenchymal tumor of the gastrointestinal tract. ${ }^{2}$ GISTs originate from the interstitial cells of Cajal, an intestinal pacemaker cell in the gut. ${ }^{3}$ GISTs most often arise in the stomach or small intestine. The presentation of GISTs varies depending on the clinical site and size. Small tumors may be detected incidentally, ${ }^{4}$ while larger tumors often present with pain, bleeding, or anemia, or bowel obstruction. ${ }^{5}$

Previously, the diagnosis of GISTs relied on tumor morphology alone, when inconsistencies and ambiguity were relatively common. ${ }^{6}$ This method of diagnosis changed after molecular characterization identified a near universal gain of function mutation in the $c$-kit gene, which encodes a proto-oncogenic tyrosine kinase, ${ }^{7}$ driving the malignant behavior of the GISTs. The KIT gene product is involved in signaling for cell survival, proliferation, and differentiation. ${ }^{8}$ GISTs not expressing the KIT mutation often have mutations in the gene encoding platelet-derived growth factor receptor alpha (PDGFRA). ${ }^{9}$ These two mutations account for approximately $90 \%$ of mutations. ${ }^{10}$ Tumors expressing either of these mutations are indistinguishable with respect to the activation of downstream signaling activation and cytogenetic changes. ${ }^{9}$ The enhanced understanding of tumor pathogenesis has dramatically changed the treatment and prognosis of GISTs, as the mutation characterization has facilitated targeted therapy with selective tyrosine kinase inhibitors (TKIs). Prior to targeted 
therapy, surgical resection was the only effective treatment for GISTs, and even resected tumors demonstrated high recurrence rates.

Imatinib, originally approved for the treatment for chronic myeloid leukemia (CML), is a small molecule TKI. The drug selectively inhibits the enzymatic activity of several tyrosine kinase receptors, including c-kit and PDGFRA. The value of imatinib in GISTs was first widely reported in 2001 with a case report showing marked, prolonged clinical response in a woman with metastatic disease. ${ }^{11}$ Since then, randomized data have supported TKI therapy in the metastatic ${ }^{12-14}$ and adjuvant setting for high-risk tumor. ${ }^{15-17}$ Imatinib is now considered standard adjuvant therapy for tumors considered at high risk for recurrence, and is used upfront for metastatic or locally advanced, unresectable tumors.

\section{Treatment in the imatinib era}

Historically, surgery was the only effective therapy, with a 5 -year survival of $42 \%$ in patients with complete resection, compared with $9 \%$ for incomplete excision. ${ }^{18}$ The majority of tumors with localized presentations could be resected, but overall outcomes were unsatisfactory due to the high risk of recurrence. ${ }^{19}$ In the pre-imatinib era, roughly $50 \%$ of patients relapsed after resection, predominantly intraabdominally, at the primary site, peritoneum, and/or liver. High-risk tumors, such as those with size greater than $5 \mathrm{~cm}$ and $>5$ mitosis/50 high-powered field (HPF), recurred more than $50 \%$ of the time within 2 years. ${ }^{19,20}$ Traditional chemotherapy did not improve outcomes for resected or recurrent disease. Thus, surgery alone did not offer long-term survival for many patients.

Significant improvements in outcomes occurred with the recognition of mutational activation of the KIT gene and the advent of TKI therapy. Randomized data support an improvement in progression-free survival (PFS) with adjuvant therapy for resected tumors $>3 \mathrm{~cm}$ in size, ${ }^{15}$ with the greatest benefit for high-risk tumors. Patients with high-risk tumors, as defined by the modified consensus criteria, consisting of tumor size $>10 \mathrm{~cm}$, mitotic count $>10 / 50 \mathrm{HPF}$, size $>5$ with mitotic rate $>5 / \mathrm{HPF}$, or tumor rupture before or at surgery, demonstrated an overall survival and recurrence free survival advantage with 36 months of imatinib. ${ }^{16}$ Currently, adjuvant imatinib is recommended for patients with a nomogram estimated risk of recurrence $>50 \%,{ }^{21}$ size $>5 \mathrm{~cm}$, mitotic count $>5 / 50 \mathrm{HPF}$, and/or with tumor rupture. ${ }^{22}$ Additional studies are ongoing to determine whether longer durations of therapy are of benefit, since trials have demonstrated high rates of recurrence shortly after discontinuation of imatinib. ${ }^{16}$ Nevertheless, median survival in the imatinib era is greatly improved, at 11.7 years in one cohort of nearly 1,200 patients. $^{23}$

Imatinib has also changed the management of metastatic or locally advanced GISTs. Neoadjuvant imatinib can make an inoperable tumor resectable, or improve the likelihood of organ preservation in some circumstances. ${ }^{24}$ For metastatic disease, survival outcomes have vastly improved. Patients with metastatic disease at presentation can now expect a median survival in excess of 6 years. ${ }^{23}$

\section{Limitations of imatinib therapy}

Despite the improvements in survival and progression observed in the imatinib era, recurrence remains a significant problem. Patterns of recurrence analysis in the American College of Surgeons Oncology Group (ACOSOG) Z9000 trial indicated a 5-year relapse free survival of $40 \%$, with local only recurrence occurring in as many as $33 \% .{ }^{25}$ Although high response rates are achieved in metastatic or advanced GISTs, complete response rates are rare, at $<10 \%$. Non-viable appearing tumor masses often contain viable cancer cells, ${ }^{26}$ suggesting that imatinib is not cytotoxic and ongoing therapy is needed to maintain the benefit. Randomized trials show a marked increase in recurrence when the drug is discontinued ${ }^{27}$ implying a "cytostatic" drug effect. High rates of recurrence noted after discontinuing the drug may suggest a delay, rather than prevention of recurrence. For example, in advanced GISTs, Le Cesne et al demonstrated that discontinuation after 3 years of continuous therapy resulted in a 2-year PFS of $16 \%{ }^{28}$ Additionally, the shape of relapse free survival curves in adjuvant trials, which drop within 2-3 years from the end of adjuvant therapy, lend further support that the increase in survival could be attributable to a delay in progression, rather than increase in cure. ${ }^{29}$

Resistance to targeted therapy is an emerging clinical problem. In the metastatic setting, the standard duration of therapy is indefinite until the development of imatinib resistance. While only $10 \%-15 \%$ of patients will have primary insensitivity to imatinib, ${ }^{30}$ secondary resistance affects $50 \%$ by 2 years. ${ }^{31}$ Therapeutic options remain limited after resistance. A minority of patients will have further response to increased doses of imatinib. ${ }^{13,32}$ Alternative TKI therapy with multi-targeted agents, such as sunitinib or regorafenib, offers low objective response rates, ${ }^{33,34}$ and improvements in PFS are of a much shorter duration. Further acquisition of mutations leading to subsequent resistance to sunitinib is a known clinical problem. ${ }^{35}$ Thus, TKI therapy and/or imatinib rechallenge are options, yet limited efficacy suggests that 
secondary resistance has created a therapeutic challenge for a significant number of GIST patients.

Tolerance of imatinib is another important consideration. Grade 3 toxicity affects as many as $25 \%-35 \%$ of patients, and includes diarrhea, muscle cramps, and edema, with increased rates of side effects reported for longer durations of therapy. Discontinuation rates, unrelated to GIST recurrence, of $26 \%$ have been reported with 36 months of adjuvant therapy. ${ }^{16}$ This is in comparison to the $13 \%$ among patients assigned to 12 months of treatment. For a therapy that may be recommended for several years, it would be of interest to investigate avenues to improve tolerability of imatinib, or reduce the overall treatment duration.

\section{Mechanisms of resistance to imatinib}

Evidence supports secondary mutations as a source of recurrent disease. ${ }^{31}$ Novel additional mutations of the KIT gene have been implicated in the development of imatinib-resistant GISTs. ${ }^{36}$ Residual microscopic clones may represent cells having acquired mutations during therapy or expression of inherent heterogeneity within the cells. Clonal heterogeneity and clonal evolution, particularly under the selection pressure of therapy, are recognized as a pattern of resistance development in many types of tumors. ${ }^{37}$ Follow-up amongst GIST patients with careful imaging demonstrate a "resistant clonal nodule" recurrence pattern in which a new enhancing focus of growth can appear within a pre-existing tumor mass in more than half of those with recurrence. ${ }^{38,39}$ Molecular analysis confirms that new mutations in KIT and PDGFRA occur in $80 \%$ of recurrent nodules biopsied. The nodule growth within the original tumor supports the idea of clonal evolution from the original tumor. Both inherent tumor heterogeneity and the acquisition of mutations could explain why a single-targeted therapy is unlikely to control disease in the long term. Development of resistance is commonly observed with chronic targeted therapy for other solid tumors, with ongoing research aimed at strategies to overcome resistance. ${ }^{40}$

Long-term imatinib therapy, which can lead to the selection of resistant clones, adds a layer of complexity to the design of further salvage therapy. Because each tumor nodule may undergo individual genetic selection of mutations in the KIT oncogene, further additional targeted therapy becomes more difficult. Alternatively, the development of locally resistant clones suggests that management with an alternative therapy, perhaps combined with imatinib, may have a role in either limiting or overcoming resistance in the ongoing management of GIST patients.

\section{The rationale for radiation therapy (RT)}

Historically, RT has not often been considered for GISTs. The gastrointestinal location and patterns of spread would potentially require large abdominal fields, presenting a narrow therapeutic window due to the dose tolerances of the small bowel to radiation. As such, there has been little investigation into the efficacy of RT.

There exist several potential scenarios where RT could provide benefit in the management of GISTs. In general, certain local therapies that are directed to resistant nodules can provide alternative avenues that do not rely on a specific molecular pathway. For example, local therapy with radiofrequency ablation has been shown to be an effective and safe treatment option for patients with limited clonal resistance. ${ }^{41,42}$ If given adjuvantly prior to failure, RT could potentially limit the development of resistance and serve as an important adjunct to imatinib. For tumors at high risk of local recurrence or R1 resection, preoperative RT could be considered, potentially with imatinib, for cytoreductive effect. A preoperative approach could allow for increased sparing of normal tissue from radiation in comparison to postoperative radiation and allow for safer dose escalation. Additionally, radiotherapy may be a local treatment option for patients with tumors that are surgically inaccessible due to location, or in whom resection would lead to severe functional impairment, such as duodenal or esophageal locations. Finally, RT can be used for palliation of local symptoms. In the setting of locally progressive and/or metastatic GISTs, short courses of RT have been shown to be effective for tumor control and symptom management, with low rates of toxicity. ${ }^{43-46}$

A number of publications provide insight into the efficacy of RT. In the largest published series, Cuaron et al reported local control in 15 of 17 treated lesions. ${ }^{45}$ Patients were most commonly treated with palliative intent after TKI failure with $30 \mathrm{~Gy}$ at $3 \mathrm{~Gy} /$ fraction, with concurrent TKIs administered in $41 \%$. The rate of partial radiographic response was $35 \%$, and the 6-month local PFS was $57 \%$. Of note, patients treated with a dose per fraction $>5 \mathrm{~Gy}(\mathrm{n}=8)$ had a radiographic response rate of $63 \%$, suggesting that GISTs are more sensitive to a higher radiation dose. Knowlton et al reported 20 years of durable local control in a 37-year-old man treated in the pre-imatinib era with debulking surgery and 36 Gy for an unresectable, nonmetastatic GIST. ${ }^{47}$ Similarly, local control was reported in six of nine patients treated with 45-60 Gy of radiation for incompletely resected disease in the pre-imatinib era. ${ }^{48}$ Radiation, when administered to a woman with progressive GIST, helped promote local tumor control to several sites of metastatic disease in tandem with other local and systemic therapies, and 
potentially prolonged survival despite disseminated disease..$^{50}$ Postoperative radiation consisting of 50.4 Gy was administered following a $\mathrm{R} 1$ resection of a $7 \mathrm{~cm}$ rectal GIST. Two years later, the patient remained free of disease. ${ }^{51}$

A few case studies have addressed concurrent radiotherapy and TKIs. Fifty Gy directed at a large supraclavicular metastasis, in combination with sorafenib, resulted in a clinical and radiographic response, and the treated tumor remained stable despite the eventual progression of other metastatic sites. ${ }^{52}$ Similarly, when radiation and imatinib were used together for the treatment of a large, incompletely resected pelvic mass, the mass regressed completely and remained locally controlled, despite the growth of a liver metastasis. ${ }^{53}$ The control of tumor at the locally treated sites, with progression at the sites of metastasis while on TKIs, suggests that radiation helped eradicate resistant clones. Radiotherapy, combined with imatinib, was effective in shrinking a large, rectal GIST, enabling a sphincter-sparing surgery without major perioperative morbidity. A pathologic complete response was achieved with a dose of $50.4 \mathrm{~Gy} .{ }^{54}$ This further supports the potential role for radiation in locally advanced GISTs that are technically unresectable.

Historically, abdominal radiation has posed technical challenges due to dose-limiting toxicities of the small bowel and visceral structures. In recent years, intensity-modulated RT (IMRT) and other technological advances have improved the therapeutic ratio of abdominal RT, allowing for dose escalation and potential reduction in acute and delayed toxicity by facilitating treatment delivery and normal tissue protection. ${ }^{55}$ Adjacent bowel does continue to limit the ability to deliver high radiation doses, yet long-term GIST control is possible with moderate doses, suggesting that treatment is feasible in a variety of circumstances. Further, simultaneous integrated boost (SIB) techniques can allow for radiation "dose painting," with lower doses at the edges of treated volumes, adjacent to critical structures. With modern advanced treatment planning, abdominal radiation is associated with mild-to-moderate acute toxicity, with a relatively low potential for serious delayed complications. ${ }^{56}$

\section{Future directions}

Local control is of paramount importance for patients with GISTs. However, in-tumor nodular recurrence, ${ }^{39}$ and resistance to imatinib, pose serious clinical challenges. Aside from the impact on overall outcomes, local failure can be associated with pain, bleeding, and other potentially threatening symptoms, for which a local treatment could be helpful. The rationale to treat a limited number of metastatic or recurrent sites of disease has been recently reviewed. ${ }^{57}$ This state of "oligometastasis" or "oligo-recurrence" may be ideal for the addition of localized therapy, potentially in combination with imatinib. As radiation is not reliant on mutation-specific responses, the addition of radiation in this setting could eradicate emergent resistant clones, while continued imatinib therapy can continue to treat sensitive clones. In this circumstance, the additional local radiotherapy could result in long-term control or cure.

Although rationale exists to support radiation in the management of GISTs, supportive evidence is limited to small case series and case reports, and additional study is needed. Future trials should address the role of radiation to either limit the development of imatinib resistance, or to eradicate limited sites of nodular local tumor growth or distant recurrence. Additionally, radiation should also be tested in the locally advanced, unresectable setting, where it may facilitate resection. Study designs should include investigation into the safety of concurrent radiotherapy with imatinib, and the ideal dose of radiotherapy for GISTs.

\section{Conclusion}

GISTs are rare intra-abdominal tumors, which are typically treated with resection and adjuvant imatinib if at high risk for recurrence, or imatinib alone if unresectable. Imatinib has prolonged the survival of patients with GISTs. However, ongoing therapy is necessary, responses are rarely complete, and the development of imatinib resistance and treatment-related toxicity are not uncommon. There are currently few durable, efficacious treatment options after imatinib failure. Radiation has an established role in the management of many other cancers for local control, and may be underutilized for GISTs, given the mechanistic rationale and support from case studies. RT has the potential to eradicate imatinib resistant clones, downstage unresectable disease, and palliate symptoms related to local progression. Further study is warranted to establish the role of RT in the multidisciplinary management of GISTs.

\section{Disclosure}

The authors report no conflicts of interest in this work.

\section{References}

1. Corless CL, Barnett CM, Heinrich MC. Gastrointestinal stromal tumours: origin and molecular oncology. Nat Rev Cancer. 2011;11(12): 865-878.

2. Miettinen M, Lasota J. Gastrointestinal stromal tumors - definition, clinical, histological, immunohistochemical, and molecular genetic features and differential diagnosis. Virchows Arch. 2001;438(1):1-12.

3. Kindblom LG, Remotti HE, Aldenborg F, Meis-Kindblom JM. Gastrointestinal pacemaker cell tumor (GIPACT): gastrointestinal stromal tumors show phenotypic characteristics of the interstitial cells of Cajal. Am J Pathol. 1998;152(5):1259-1269. 
4. Burkill GJ, Badran M, Al-Muderis O, et al. Malignant gastrointestinal stromal tumor: distribution, imaging features, and pattern of metastatic spread. Radiology. 2003;226(2):527-532.

5. Chou FF, Eng HL, Sheen-Chen SM. Smooth muscle tumors of the gastrointestinal tract: analysis of prognostic factors. Surgery. 1996;119(2):171-177.

6. Newman PL, Wadden C, Fletcher CD. Gastrointestinal stromal tumours: correlation of immunophenotype with clinicopathological features. J Pathol. 1991;164(2):107-117.

7. Hirota S, Isozaki K, Moriyama Y, et al. Gain-of-function mutations of c-kit in human gastrointestinal stromal tumors. Science. 1998;279(5350): 577-580.

8. Edling CE, Hallberg B. c-Kit - a hematopoietic cell essential receptor tyrosine kinase. Int J Biochem Cell Biol. 2007;39(11):1995-1998.

9. Heinrich MC, Corless CL, Duensing A, et al. PDGFRA activating mutations in gastrointestinal stromal tumors. Science. 2003;299(5607): $708-710$.

10. Joensuu H. Gastrointestinal stromal tumor (GIST). Ann Oncol. 2006; 17 Suppl 10:x280-x286.

11. Joensuu H, Roberts PJ, Sarlomo-Rikala M, et al. Effect of the tyrosine kinase inhibitor STI571 in a patient with a metastatic gastrointestinal stromal tumor. N Engl J Med. 2001;344(14):1052-1056.

12. Demetri GD, von Mehren M, Blanke CD, et al. Efficacy and safety of imatinib mesylate in advanced gastrointestinal stromal tumors. $N$ Engl J Med. 2002;347(7):472-480.

13. Blanke CD, Rankin C, Demetri GD, et al. Phase III randomized, intergroup trial assessing imatinib mesylate at two dose levels in patients with unresectable or metastatic gastrointestinal stromal tumors expressing the kit receptor tyrosine kinase: S0033. J Clin Oncol. 2008;26(4): 626-632.

14. Verweij J, Casali PG, Zalcberg J, et al. Progression-free survival in gastrointestinal stromal tumours with high-dose imatinib: randomised trial. Lancet. 2004;364(9440):1127-1134.

15. Dematteo RP, Ballman KV, Antonescu CR, et al; American College of Surgeons Oncology Group (ACOSOG) Intergroup Adjuvant GIST Study Team. Adjuvant imatinib mesylate after resection of localised, primary gastrointestinal stromal tumour: a randomised, double-blind, placebo-controlled trial. Lancet. 2009;373(9669):1097-1104.

16. Joensuu H, Eriksson M, Sundby Hall K, et al. One vs three years of adjuvant imatinib for operable gastrointestinal stromal tumor: a randomized trial. JAMA. 2012;307(12):1265-1272.

17. Casali PG, Le Cesne A, Poveda Velasco A, et al. Imatinib failure-free survival (IFS) in patients with localized gastrointestinal stromal tumors (GIST) treated with adjuvant imatinib (IM): the EORTC/AGITG/FSG/ GEIS/ISG randomized controlled phase III trial. J Clin Oncol. 2013; 31 Suppl: abst 10500.

18. Pierie JP, Choudry U, Muzikansky A, Yeap BY, Souba WW, Ott MJ. The effect of surgery and grade on outcome of gastrointestinal stromal tumors. Arch Surg. 2001;136(4):383-389.

19. DeMatteo RP, Lewis JJ, Leung D, Mudan SS, Woodruff JM, Brennan MF. Two hundred gastrointestinal stromal tumors: recurrence patterns and prognostic factors for survival. Ann Surg. 2000;231(1):51-58.

20. Nilsson B, Bumming P, Meis-Kindblom JM, et al. Gastrointestinal stromal tumors: the incidence, prevalence, clinical course, and prognostication in the preimatinib mesylate era - a population-based study in western Sweden. Cancer. 2005;103(4):821-829.

21. Gold JS, Gönen M, Gutiérrez A, et al. Development and validation of a prognostic nomogram for recurrence-free survival after complete surgical resection of localised primary gastrointestinal stromal tumour: a retrospective analysis. Lancet Oncol. 2009;10(11):1045-1052.

22. National Comprehensive Cancer Network. Soft Tissue Sarcoma. Version I. Fort Washington, PA: National Comprehensive Cancer Network; 2014 Available from: http://www.nccn.org/professionals/physician_gls/pdf/ sarcoma.pdf. Accessed April 15, 2014

23. Call J, Walentas CD, Eickhoff JC, Scherzer N. Survival of gastrointestinal stromal tumor patients in the imatinib era: life raft group observational registry. BMC Cancer. 2012;12:90.
24. Eisenberg BL, Harris J, Blanke CD, et al. Phase II trial of neoadjuvant/ adjuvant imatinib mesylate (IM) for advanced primary and metastatic/ recurrent operable gastrointestinal stromal tumor (GIST): early results of RTOG 0132/ACRIN 6665. J Surg Oncol. 2009;99(1):42-47.

25. DeMatteo RP, Ballman KV, Antonescu CR, et al; American College of Surgeons Oncology Group (ACOSOG) Intergroup Adjuvant GIST Study Team for the Alliance for Clinical Trials in Oncology. Long-term results of adjuvant imatinib mesylate in localized, high-risk, primary gastrointestinal stromal tumor: ACOSOG Z9000 (Alliance) intergroup phase 2 trial. Ann Surg. 2013;258(3):422-429.

26. Bauer S, Hartmann JT, de Wit M, et al. Resection of residual disease in patients with metastatic gastrointestinal stromal tumors responding to treatment with imatinib. Int J Cancer. 2005;117(2):316-325.

27. Blay JY, Le Cesne A, Ray-Coquard I, et al. Prospective multicentric randomized phase III study of imatinib in patients with advanced gastrointestinal stromal tumors comparing interruption versus continuation of treatment beyond 1 year: the French Sarcoma Group. J Clin Oncol. 2007;25(9):1107-1113.

28. Le Cesne A, Ray-Coquard I, Bui BN, et al. Discontinuation of imatinib in patients with advanced gastrointestinal stromal tumours after 3 years of treatment: an open-label multicentre randomised phase 3 trial. Lancet Oncol. 2010;11(10):942-949.

29. Casali PG, Fumagalli E, Gronchi A. Adjuvant therapy of gastrointestinal stromal tumors (GIST). Curr Treat Options Oncol. 2012;13(3): 277-284.

30. Van Glabbeke M, Verweij J, Casali PG, et al. Initial and late resistance to imatinib in advanced gastrointestinal stromal tumors are predicted by different prognostic factors: a European Organisation for Research and Treatment of Cancer-Italian Sarcoma Group-Australasian Gastrointestinal Trials Group study. J Clin Oncol. 2005;23(24): 5795-5804.

31. Antonescu CR, Besmer P, Guo T, et al. Acquired resistance to imatinib in gastrointestinal stromal tumor occurs through secondary gene mutation. Clin Cancer Res. 2005;11(11):4182-4190.

32. Zalcberg JR, Verweij J, Casali PG, et al. Outcome of patients with advanced gastro-intestinal stromal tumours crossing over to a daily imatinib dose of $800 \mathrm{mg}$ after progression on $400 \mathrm{mg}$. Eur J Cancer. 2005;41(12):1751-1757.

33. Demetri GD, Garrett CR, Schöffski P, et al. Complete longitudinal analyses of the randomized, placebo-controlled, phase III trial of sunitinib in patients with gastrointestinal stromal tumor following imatinib failure. Clin Cancer Res. 2012;18(11):3170-3179.

34. Demetri GD, Reichardt P, Kang YK, et al. Efficacy and safety of regorafenib for advanced gastrointestinal stromal tumours after failure of imatinib and sunitinib (GRID): an international, multicentre, randomised, placebo-controlled, phase 3 trial. Lancet. 2013;381(9863):295-302.

35. Guo T, Hajdu M, Agaram NP, et al. Mechanisms of sunitinib resistance in gastrointestinal stromal tumors harboring KITAY502-3ins mutation: an in vitro mutagenesis screen for drug resistance. Clin Cancer Res. 2009;15(22):6862-6870.

36. Wang CM, Huang K, Zhou Y, et al. Molecular mechanisms of secondary imatinib resistance in patients with gastrointestinal stromal tumors. $J$ Cancer Res Clin Oncol. 2010;136(7):1065-1071.

37. Aparicio S, Caldas C. The implications of clonal genome evolution for cancer medicine. N Engl J Med. 2013;368(9):842-851.

38. Desai J, Shankar S, Heinrich MC, et al. Clonal evolution of resistance to imatinib in patients with metastatic gastrointestinal stromal tumors. Clin Cancer Res. 2007;13(18 Pt 1):5398-5405.

39. Shankar S, vanSonnenberg E, Desai J, Dipiro PJ, Van Den Abbeele A, Demetri GD. Gastrointestinal stromal tumor: new nodule-within-amass pattern of recurrence after partial response to imatinib mesylate. Radiology. 2005;235(3):892-898.

40. Chong CR, Jänne PA. The quest to overcome resistance to EGFRtargeted therapies in cancer. Nat Med. 2013;19(11):1389-1400.

41. Jones RL, McCall J, Adam A, et al. Radiofrequency ablation is a feasible therapeutic option in the multi modality management of sarcoma. Eur J Surg Oncol. 2010;36(5):477-482. 
42. Dileo P, Randhawa R, Vansonnenberg E, et al. Safety and efficacy of percutaneous radio-frequency ablation (RFA) in patients (pts) with metastatic gastrointestinal stromal tumor (GIST) with clonal evolution of lesions refractory to imatinib mesylate (IM). J Clin Oncol. 2004;22(Suppl 14): abst 9024.

43. Di Scioscio V, Greco L, Pallotti MC, et al. Three cases of bone metastases in patients with gastrointestinal stromal tumors. Rare Tumors. 2011;3(2):e17.

44. Tezcan Y, Koç M. Gastrointestinal stromal tumor of the rectum with bone and liver metastasis: a case study. Med Oncol. 2011;28 Suppl 1: S204-S206.

45. Cuaron JJ, Goodman KA, Lee N, Wu AJ. External beam radiation therapy for locally advanced and metastatic gastrointestinal stromal tumors. Radiat Oncol. 2013;8(1):274.

46. Hurwitz J, Constantinidou A, Saran F, et al. The role of radiotherapy in metastatic gastrointestinal stromal tumor (GIST). Proc Connect Tissue Oncol Soc. 2008(2008): abst 35023.

47. Knowlton CA, Brady LW, Heintzelman RC. Radiotherapy in the treatment of gastrointestinal stromal tumor. Rare Tumors. 2011;3(4):e35.

48. Crosby JA, Catton CN, Davis A, et al. Malignant gastrointestinal stromal tumors of the small intestine: a review of 50 cases from a prospective database. Ann Surg Oncol. 2001;8(1):50-59.

49. Drazin D, Spitler K, Jeswani S, Shirzadi A, Bannykh S, Patil C. Multiple intracranial metastases from a gastric gastrointestinal stromal tumor. J Clin Neurosci. 2013;20(3):471-473.

50. Chiguchi G, Kawana K, Nagase H, et al. [A case of small intestinal stage IV gastrointestinal stromal tumor in which long-term disease control was maintained for more than 10 years through a multidisciplinary team approach]. Gan To Kagaku Ryoho. [Cancer Chemotherapy]. 2012;39(12):1898-1901. Japanese.
51. Pollock J, Morgan D, Denobile J, Williams J. Adjuvant radiotherapy for gastrointestinal stromal tumor of the rectum. Dig Dis Sci. 2001;46(2): 268-272.

52. Lolli C, Pantaleo MA, Nannini M, et al. Successful radiotherapy for local control of progressively increasing metastasis of gastrointestinal stromal tumor. Rare Tumors. 2011;3(4):e49.

53. Boruban C, Sencan O, Akmansu M, Atik ET, Ozbek S. Metastatic gastrointestinal stromal tumor with long-term response after treatment with concomitant radiotherapy and imatinib mesylate. Anticancer Drugs. 2007;18(8):969-972.

54. Ciresa M, D'Angelillo RM, Ramella S, et al. Molecularly targeted therapy and radiotherapy in the management of localized gastrointestinal stromal tumor (GIST) of the rectum: a case report. Tumori. 2009;95(2): 236-239.

55. Taremi M, Ringash J, Dawson LA. Upper abdominal malignancies: intensity-modulated radiation therapy. Front Radiat Ther Oncol. 2007;40:272-288.

56. Nour AA, Alaradi A, Mohamed A, Altuwaijri S, Rudat V. Intensity modulated radiotherapy of upper abdominal malignancies: dosimetric comparison with 3D conformal radiotherapy and acute toxicity. Radiat Oncol. 2013;8(1):207.

57. Corbin KS, Hellman S, Weichselbaum RR. Extracranial oligometastases: a subset of metastases curable with stereotactic radiotherapy. J Clin Oncol. 2013;31(11):1384-1390.
OncoTargets and Therapy

\section{Publish your work in this journal}

OncoTargets and Therapy is an international, peer-reviewed, open access journal focusing on the pathological basis of all cancers, potential targets for therapy and treatment protocols employed to improve the management of cancer patients. The journal also focuses on the impact of management programs and new therapeutic agents and protocols on

\section{Dovepress}

patient perspectives such as quality of life, adherence and satisfaction The manuscript management system is completely online and includes a very quick and fair peer-review system, which is all easy to use. Visit http://www.dovepress.com/testimonials.php to read real quotes from published authors. 